\title{
MIRADAS A LAS ALTERNATIVAS EDUCATIVAS AL FRACASO ESCOLAR. POLÍTICAS, EXPERIENCIAS, CONCEPTUALIZACIONES Y ESTUDIO DE CASO A INICIOS DEL SXXI
}

\author{
Cecilia Durantini Villarino* \\ Universidad de Buenos Aires, Argentina \\ ceciliadurantini@gmail.com
}

Recibido: 3/10/2017 Aceptado: 14/10/2018

\section{Resumen}

Las últimas décadas del SXX en América Latina están signadas por las secuelas del autoritarismo y la fragmentación neoliberal. La primera década del SXXI transcurrió con cambios favorables en la vida social. Las políticas para la justicia, igualdad e inclusión educativas representan una tendencia con logros efectivos en la materialización del derecho a la educación. No obstante, sus límites en la superación de las brechas en el acceso y calidad de los aprendizajes. Las alternativas educativas en respuesta al fracaso escolar se diversificaron y si bien se cuenta con estudios en el campo, resta profundizar sobre sus condiciones, funcionamiento cotidiano, resultados e impacto en los distintos niveles de gestión.

Aquí se recuperan aportes institucionales para pensar las fronteras que las organizaciones imponen a lo posible y las que estas alternativas movilizan. Luego se exponen los resultados de un estudio de caso sobre una alternativa para la reinserción con foco en las posibilidades y dificultades asociadas a la ubicación en el sistema educativo, la inserción en el barrio y la articulación con la escuela.

Las alternativas apuestan al cambio para la democratización educativa. Al hacerlo develan los mecanismos y prácticas productores de exclusión. Lo cual compromete aún más la pregunta por su potencial y el de las instituciones actuales para garantizar el cumplimiento del derecho a una educación de calidad para todos.

Palabras clave: Alternativas educativas - Fracaso escolar - Articulación institucional - Inserción territorial - Ubicación organizacional.

* Es Doctora en Educación, Facultad de Filosofía y Letras. Universidad de Buenos Aires. Licenciada en Ciencias de la Educación, diploma de honor, Facultad de Filosofía y Letras. UBA. Profesora para la Enseñanza Primaria, Escuela Normal Superior $\mathrm{N}^{\circ} 5$. Ciudad de Buenos Aires. Ayudante Docente de Primera, Teorías Psicológicas de la Subjetividad, Licenciatura Ciencias de la Educación, Facultad de Filosofía y Letras. UBA. 
Revista de la Escuela de Ciencias de la Educación, año 14, nRo. 13, vol. 2, Julio a diciembre de 2018. Páginas $121-141$. ISSN 1851-6297 (DESDE DICIEMBRE DE 2006 A DICIEMBRE DE 2017). ISSN 2362-3349 (EN LíNEA). MIRADAS A LAS ALTERNATIVAS EDUCATIVAS AL FRACASO ESCOLAR. POLITICAS, EXPERIENCIAS, CONCEPTUALIZACIONES Y ESTUDIO DE CASO A INICIOS DEL $S$ XXI. Cecilia Durantini VilLaRino.

\section{Abstract}

The last decades of the SXX in Latin America are marked by the aftermath of authoritarianism and fragmentation. The first decade of the SXXI passed with favorable changes in social life. Policies for justice, equality and inclusion in education represent a trend with effective achievements in the realization of the right to education, notwithstanding its limitations.

Although there are studies on proposals in response to school failure, there is a deepening of the favorable conditions and limits to overcome it, results obtained and impact at the different levels of management.

Here we introduce contributions of institutional perspectives to continue thinking the borders that organizations impose to the possible in the educational field and those that the alternatives mobilize. The results of the study carried out on an alternative for reintegration are presented, focusing on the possibilities and difficulties associated with placement in the educational system, insertion in the neighborhood and articulation with the school.

Alternatives bet on the change for inclusion. They also reveal exclusionary mechanisms and practices. This further compromises the question of its potential and that of the current institutions to guarantee the fulfillment of the right to a quality education for all.

Keywords: Educational alternatives - School failure - Institutional articulation - Territorial insertion - Organizational location.

\section{Notas sobre Políticas y Fracaso escolar al inicio del SXXI}

Las últimas décadas del SXX en América Latina están signadas por las secuelas del autoritarismo y la fragmentación de las dictaduras militares; la inestabilidad política y las crisis económicas de los '80 y el aumento de la desigualdad y la desprotección social del neoliberalismo de los ' 90 . Este escenario impuso el problema sobre el sentido de las acciones educativas ante un contexto de emergencia social que afectaba al conjunto de los actores (Tedesco, 2012).

La primera década del SXXI transcurrió con cambios favorables en la vida social, alentados por la continuidad democrática, la consolidación de los poderes públicos y el crecimiento económico de la región. No obstante la ampliación de los derechos económicos y sociales, el nivel de pobreza, la marcada desigualdad y baja movilidad regional continúan siendo alarmantes (Ferreira, 2013).

En el campo educativo, aunque se avanzó en la universalización, permanencia y extensión de la obligatoriedad (Tenti Fanfani, 2001) la superación de las desigualdades sigue limitada por las condiciones socio económicas que dificultan la escolaridad a los grupos más vulnerables. Existe consenso en considerar este problema desde un continuo exclusión-inclusión que contem- 
Revista de la Escuela de Ciencias de la Educación, año 14, nRo. 13, vol. 2, Julio a diciembre de 2018. Páginas 121-141. ISSN 1851-6297 (DESDE DICIEMBRE DE 2006 A DICIEMBRE DE 2017). ISSN 2362-3349 (EN LiNEA). MiRADAS A LAS ALTERNAtiVAS EDUCATIVAS AL FRACASO ESCOLAR. POLITICAS, EXPERIENCIAS, CONCEPTUALIZACIONES Y ESTUdIO DE CASO A INICIOS DEL S XXI. Cecilia Durantini Villarino.

ple la equidad en el acceso, permanencia y egreso, los logros de aprendizaje y la relevancia, calidad y pertinencia de los saberes (Terigi, 2009; Poggi, 2014).

Las políticas de democratización educativa representan una tendencia distintiva del período (Tedesco, 2012; UNESCO-OREALC, 2008) con impacto positivo a pesar de sus limitaciones.

Rivas (2015) describe un proceso de expansión de los derechos educativos dado por la ampliación del acceso, el incremento del financiamiento estatal y el reconocimiento de los derechos de poblaciones excluidas mediante la diversificación de las políticas educativas. Entre las tendencias dominantes, la justicia educativa como estrategia contra la desigualdad social se expresó en los programas de transferencias condicionadas, la apertura de escuelas, la profundización de las políticas compensatorias y los planes de mejora para las escuelas más vulnerables. También en experiencias como flexibilización de los regímenes académicos, renovación de la autonomía escolar y extensión del tiempo sin modificaciones significativas al formato establecido o derivadas hacia cambios más profundos.

Para Tenti Fanfani (2007) se superaron los límites de las medidas compensatorias gracias a la diversificación de la oferta pedagógica y el fortalecimiento de las iniciativas institucionales en adecuación al territorio. Aunque advierte sobre las desarticulaciones entre el diseño de los programas y la aplicación concreta, y los efectos perversos como la estigmatización de los destinatarios, la condescendencia pedagógica y la escuela "sobrecargada" de funciones pero subdotada de recursos. Bajo este escenario, visualiza dos alternativas. Si la escuela se mantiene como agencia especializada en la función pedagógica, deberá articular óptimamente con los servicios a cargo de las condiciones sociales del aprendizaje. Si se convierte en una agencia de desarrollo integral de la infancia, será preciso diseñar otra institución.

Las observaciones y proyecciones expuestas adquieren particular interés frente al escenario actual. Aquí contextualizan el análisis de las respuestas al fracaso escolar centradas en el cambio organizacional.

\section{Experiencias ante el fracaso escolar}

En la investigación doctoral (Durantini Villarino, 2014) sobre respuestas centradas en el cambio organizacional para recuperar la continuidad de las trayectorias y aprendizajes de niños y niñas fuera del sistema o con vivencias reiteradas de fracaso, se recopilan relatos directos de experiencias en grupos no graduados, redistribuciones del tiempo escolar, modalidades participativas de evaluación y promoción, definición de nuevas figuras docentes y encuadres de inserción comunitaria. Asimismo, se recogen estudios y evaluaciones que aportan dimensiones, perspectivas y herramientas propias para la sistematización y el análisis de este tipo de propuestas. 
Revista de la Escuela de Ciencias de la Educación, año 14, nRo. 13, vol. 2, Julio a diciembre de 2018. Páginas $121-141$. ISSN 1851-6297 (DESDE DICIEMBRE DE 2006 A DICIEMBRE DE 2017). ISSN 2362-3349 (EN LíNEA). MIRADAS A LAS ALTERNATIVAS EDUCATIVAS AL FRACASO ESCOLAR. POLITICAS, EXPERIENCIAS, CONCEPTUALIZACIONES Y ESTUdIO DE CASO A INICIOS DEL $S$ XXI. Cecilia Durantini VilLaRino.

Ambas fuentes nutren la interrogación por el sentido de la escuela y los horizontes del cambio en un contexto de "declive de la institución escolar moderna" (Dubet, 2002). Baquero (2006) apuesta al conocimiento de las alternativas al formato clásico, exitosas con poblaciones vulnerabilizadas, para estimar las potencialidades de la acción educativa frente a la singularidad de los alumnos y las situaciones escolares. Si bien se cuenta con contribuciones de este tipo (Padawer, 2009; Terigi, 2013) resta profundizar sobre los resultados obtenidos en responder al fracaso escolar y su impacto en los componentes asociados al fracaso en los distintos niveles de gestión para replantear diferentes cuestiones. Particularmente, el tema del cambio, sus límites y posibilidades en las particulares configuraciones de poder. No sería la primera vez en la historia del sistema, que los contenidos más transformadores de las alternativas quedan neutralizados bajo los modelos dominantes (Puiggrós, 1990), las iniciativas democratizadoras recuperadas por las autoridades (Lobrot, 1974, p.54) y las experiencias interrumpidas y apartados sus fundadores (Trilla, 2005).

\section{Aproximaciones institucionales}

Para continuar pensando las fronteras que las organizaciones imponen a lo posible en el campo educativo y las que las alternativas movilizan, se introducen ahora aportes institucionales.

Castoriadis (2007) define la institución como una red simbólica cuyo componente funcional, ligado a la satisfacción de una necesidad social se combina con el imaginario. Lo imaginario remite a un estructurante estructurado, un significado-significante central que instaura las significaciones con las que los sujetos captan la realidad y el sentido impuesto a las relaciones entre ellos, los actos y los objetos a nivel práctico, afectivo y mental. Su carácter compartido es condición de existencia de la institución bajo un modo de existir propio en su tiempo histórico y a cada momento. A nivel individual, opera como un esquema organizador-organizado del mundo, que constituye al sujeto en su singularidad. A nivel social, existe como las significaciones sociales imaginarias y como la imaginación radical creadora de esas significaciones. El nivel de alienación social respecto al reconocimiento de las significaciones y materializaciones establecidas como productos de la propia actividad instituyente marca el horizonte a la imaginación de nuevas definiciones, figuras y herramientas de vida para un colectivo en un momento dado de su historia.

Bajo esta clave, las alternativas al formato escolar convencional representan un ejemplo de lo instituyente en el campo de la educación. En tanto productos sociales del período ponen en escena otras respuestas acerca de quiénes el colectivo, qué son unos para otros, dónde y en qué están, qué desean y qué hace falta. Con lo cual interpelan las categorías desde las que se piensa 
Revista de la Escuela de Ciencias de la Educación, año 14, nRo. 13, vol. 2, Julio a diciembre de 2018. Páginas 121-141. ISSN 1851-6297 (DESDE DICIEMBRE DE 2006 A DICIEMBRE DE 2017). ISSN 2362-3349 (EN LiNEA). MiRADAS A LAS ALTERNAtiVAS EDUCATIVAS AL FRACASO ESCOLAR. POLITICAS, EXPERIENCIAS, CONCEPTUALIZACIONES Y ESTUdIO DE CASO A INICIOS DEL S XXI. Cecilia Durantini Villarino.

lo común y se articulan con las versiones que otros colectivos y grupos dan a estas mismas cuestiones. Los derroteros que siguen vendrían a describir la potencia y los recursos de los grupos y sujetos para generar nuevas formas de lo educativo ante las fronteras impuestas, en las distintas dimensiones de la vida social, a la materialización de un proyecto y hacer diferentes.

Enríquez $(1992,2002)$ permite continuar esta línea de interrogantes a partir de definir la organización como un sistema cultural, simbólico e imaginario que articula la instancia mítica, socio-histórica, institucional y organizacional (strictu sensu). En la instancia institucional convergen el amor que vincula a los sujetos entre sí y la violencia ejercida contra lo otro. Esta instancia proporciona la misión, las orientaciones y los valores que la organizacional interpreta y estructura bajo una modalidad específica y transitoria. Así la necesidad institucional de estabilizar las conductas se traduce en la división del trabajo; el problema del poder en los sistemas de autoridad; la canalización de los deseos en el proyecto y en las condiciones de desempeño. Con lo cual, la instancia organizacional da existencia a la institucional y la expresa o la deforma en el cumplimiento de su tarea principal mediante procesos organizativos de estructuración.

Estos procesos defienden contra las angustias fundamentales que la instancia institucional activa. Una es el miedo a lo informe ligado al origen, a lo espontáneo y al movimiento creador, contra el cual la organización opone la estructura. Contra el miedo a las pulsiones destructivas, la organización implementa sistemas de normas que limitan la competencia interna o de recompensas que la exaltan. Mientras que el miedo a la pulsión de vida, se controla por un lado, canalizando esta pulsión en el trabajo productivo, la cohesión para realizarlo y la admiración hacia los dirigentes, y por el otro, inhibiendo las expresiones de afecto entre los miembros. Frente al miedo a lo desconocido y a lo nuevo, se aplican métodos supuestamente racionales de toma de decisiones. El miedo a los otros, que activa el fantasma de la guerra de todos contra todos, conduce al refuerzo de la cohesión o el énfasis en la asignación diferencial de funciones y responsabilidades. El miedo a la palabra libre, no prevista, que inquieta por su potencial desviador, despierta la sospecha y la resistencia ante los proyectos innovadores. Por último, contra el miedo al pensamiento inventivo, la reflexión y la interrogación que pueden tomar como objeto los valores y los modos de entender dominantes, la organización impone la represión de la creatividad. Por consiguiente, la instancia organizacional se vincula fundamentalmente a la repetición, manifiesta en la conformidad y la ritualización, antes que con el cambio y en ese sentido, funciona siguiendo un modelo preciso y no un proceso vivo.

La tesis de Enríquez permite conjeturar que las alternativas revisten un alto potencial para despertar el tipo de angustias referidas. En este marco, las dificultades y limitaciones que les opone el sistema educativo en general 
Revista de la Escuela de Ciencias de la Educación, año 14, nRo. 13, vol. 2, Julio a diciembre de 2018. Páginas $121-141$. ISSN 1851-6297 (DESDE DICIEMBRE DE 2006 A DICIEMBRE DE 2017). ISSN 2362-3349 (EN LínEA). MIRADAS A LAS ALTERNATIVAS EDUCATIVAS AL FRACASO ESCOLAR. POLITICAS, EXPERIENCIAS, CONCEPTUALIZACIONES Y ESTUDIO DE CASO A INICIOS DEL $S$ XXI. Cecilia Durantini VilLaRino.

y cada escuela en particular, deberían leerse como manifestaciones reactivas contra aquellas. En la misma línea, el proyecto, los modelos e ideología de las alternativas informarían sobre los contenidos singulares que adquieren en ellas la misión, las orientaciones y los valores de la institucional. A la vez que las formas organizacionales que asumen arrojarían luz sobre los modos que asumen esos miedos al interior de las alternativas mismas.

Dubet (2002) identifica a la escuela como una forma de trabajo sobre los otros que debía conciliar las contradicciones del proyecto moderno entre la integración en una cultura común, la jerarquización social y el reconocimiento de la singularidad y la autorrealización. En respuesta a lo cual adecuó las estrategias de socialización, enseñanza y formación de la personalidad desplegadas a lo largo del tiempo. A la vez que se estabilizaba como una estructura simbólica organizadora de la acción, las relaciones y el sentido de las experiencias de los individuos en ella. Las críticas actuales a la escuela, la complejidad de las exigencias que se le dirigen y el ritmo acelerado de las innovaciones pedagógicas estarían revelando la exacerbación de las contradicciones latentes de la modernidad. Dubet lee un declive institucional que compromete los principios de universalidad, mediación desde un centro e igualdad, y expone a los individuos a desigualdades que amplifican la experiencia de sufrimiento, desprecio y distancia de los otros. Este diagnóstico interroga a las alternativas educativas como expresiones del declive y a la singularidad de las estrategias que generan frente a las lógicas en tensión, entre la integración, adquisición de competencias y desarrollo individual y las respectivas lógicas de socialización, enseñanza y formación de la persona.

Los aportes institucionales referenciados abren en su conjunto a la cuestión de cuáles modos de orientación y articulación con la sociedad proyectan las alternativas, qué tipo de experiencia social se proponen generar, mediante cuáles estrategias y cuál es su impacto en la vida de los sujetos y las instituciones. ¿Cuál es su potencial para posibilitar una ciudadanía responsable, el acceso a los conocimientos y el desarrollo pleno de la subjetividad? ¿Los cambios que desarrollan descubren las limitaciones de la escuela para generar propuestas creativas ante las condiciones actuales? ¿Logran construir formatos que contribuyan a una relación más dinámica con el contexto que la escolar? ¿Qué sentidos les atribuyen los protagonistas, los otros actores del sistema y de la sociedad en su conjunto, a las alternativas educativas como respuestas al fracaso escolar?

\section{Mirada desde un estudio de caso}

La interrogación sobre las alternativas educativas sigue con los resultados de la investigación doctoral (Durantini Villarino, 2014), que se propuso identificar las posibilidades y limitaciones de este tipo de respuestas al fracaso escolar. En esta investigación, las alternativas se definen como aquellas pro- 
Revista de la Escuela de Ciencias de la Educación, año 14, nRo. 13, vol. 2, Julio a diciembre de 2018. Páginas 121-141. ISSN 1851-6297 (DESDE DICIEMBRE DE 2006 A DICIEMBRE DE 2017). ISSN 2362-3349 (EN LiNEA). MiRADAS A LAS ALTERNATIVAS EDUCATIVAS AL FRACASO ESCOLAR. POLITICAS, EXPERIENCIAS, CONCEPTUALIZACIONES Y ESTUDIO DE CASO A INICIOS DEL $S$ XXI. Cecilia Durantini Villarino.

puestas ubicadas dentro del sistema que presentan condiciones diferentes a las escolares con respecto a la idiosincrasia (Fernández, 1994) y la normativa de las organizaciones de la modalidad y el nivel de referencia, bajo el supuesto de su incidencia favorable en el aprendizaje de niños y niñas con reiteradas experiencias de fracaso escolar.

Esta investigación se inscribe en la línea de estudios sobre dinámicas institucionales en situaciones críticas dirigida por Lidia Fernández desde 1988 bajo el Programa "Instituciones educativas" (IICE-UBA). En el año 2003, se exploran proyectos generados ante situaciones críticas (Durantini Villarino, 2004). En la investigación doctoral, se profundizan las posibilidades y los límites de las alternativas educativas dentro del sistema formal a partir de un estudio de caso. El cual se ha seleccionado por referencias de informantes clave, exploraciones previas y recopilaciones de experiencias implementadas en América Latina. El caso se toma como ejemplo que define la inteligibilidad del conjunto al cual pertenece y constituye como fenómeno único, su aporte no deriva de la generalización o el grado de espectacularidad que reviste sino de su valor heurístico como variación ejemplar (Agamben, 2003).

El estudio del caso reconstruye la combinatoria de condiciones implicadas en el despliegue de procesos de inclusión social y oportunidades de vivir experiencias de aprendizaje positivas en el grupo. Su continuidad en el tiempo jugó un papel clave para el desarrollo de la confianza y la autonomía en estos niños que habían sufrido reiterados fracasos escolares. Los logros se vieron limitados por diferentes factores, de los cuales aquí interesa comunicar en particular los fenómenos de oposición al cambio.

\section{Contextos del caso}

El actual Programa Grados de Nivelación (en adelante, PGN) constituye una de las respuestas al problema del fracaso escolar que ofrece la Ciudad de Buenos Aires. Sus destinatarios son los niños de 8 a 14 años que nunca asistieron o abandonaron la escuela primaria. La apertura de Grados de Nivelación (en adelante, GN) se propone como "puente de acceso" para "nivelar" educativamente a estos niños en el menor tiempo posible e integrarlos al grado más próximo a su edad, garantizando los aprendizajes básicos del nivel primario común. Para lograrlo, PGN apuesta a una estructura no graduada de grupos pequeños, formas individualizadas de enseñanza y evaluación y seguimiento y apoyo a los niños integrados en los grados comunes.

Cada GN depende de un establecimiento, los niños son sus alumnos y se incluyen en actividades con los grados comunes. No obstante, pueden situarse dentro o fuera del edificio, para facilitar la continuidad de los niños que manifiestan mayor resistencia hacia la escuela. 
Revista de la Escuela de Ciencias de la Educación, año 14, nRo. 13, vol. 2, Julio a diciembre de 2018. Páginas $121-141$. ISSN 1851-6297 (DESDE DICIEMBRE DE 2006 A DICIEMBRE DE 2017). ISSN 2362-3349 (EN LíNEA). MIRADAS A LAS ALTERNATIVAS EDUCATIVAS AL FRACASO ESCOLAR. POLITICAS, EXPERIENCIAS, CONCEPTUALIZACIONES Y ESTUdIO DE CASO A INICIOS DEL $S$ XXI. Cecilia Durantini VilLaRino.

Los GN no siguen un programa ni didácticas determinados, sino que los maestros efectúan adecuaciones grupales e individuales. Se acentúa la adquisición de los hábitos y normas escolares, la mirada y escucha atenta a cada niño y el acompañamiento a los recorridos y las situaciones personales. La permanencia máxima en los GN es de dos años. Para evitar un circuito terminal, el niño retorna progresivamente al grado común determinado según su nivel de aprendizaje, edad y personalidad y las características de los grados de destino.

El equipo de PGN está conformado por un coordinador, capacitadores, asistentes técnico-pedagógicos para los GN, y maestros comunes a cargo de los mismos. La matrícula se compone con niños que ingresaron al GN en algún momento del año, completaron el ciclo lectivo allí o retornaron al grado común en el transcurso del mismo. Se trata de niños con trayectorias vitales y escolares fragmentadas en situaciones de alta vulnerabilización social y económica.

La historia de PGN comienza en 1999 por iniciativa de un grupo de directores, maestros y referentes sanitarios y barriales de una de las zonas más pobres de la ciudad, ante el encuentro con niños que, en edad teórica escolar, no estaban en la escuela primaria. En el año 2000 se abren los primeros GN dentro de un establecimiento educativo. Al año siguiente, bajo el supuesto de que la aproximación a los contextos de referencia para los niños puede contribuir a su permanencia, abren un GN dentro de un centro comunitario. En el año 2002, los GN se incorporan al Programa Zona de Acción Prioritaria de la Secretaría de Educación.

Al año siguiente, un asistente técnico asume la coordinación y promueve la oficialización del proyecto concretada en 2003 bajo la Dirección de Nivel Primario por Resolución 2429/03 del Ministerio de Educación de la Ciudad. Para el coordinador, este hecho marca un hito pues la normativa permite evitar la derivación de alumnos desde la escuela a los GN y su funcionamiento como instancia terminal. La ubicación de PGN dentro de la Dirección de Primaria impulsa a delinear un perfil propio. Desde el año 2010, el proyecto depende de la Coordinación de Inclusión Educativa correspondiente a la Dirección de Inclusión. Estas modificaciones no se actualizan en la normativa.

La evolución de PGN muestra una proyección creciente en el alcance sobre la población destinataria, la cobertura territorial y la oferta educativa. En el año 2000, de los 2.245 niños de 6 a 12 años no escolarizados en la Ciudad, el primer GN recibe 21 niños, 6 pasaron a un grado común y 15 desertaron. En el año 2010 , el $60 \%$ de los inscriptos se incorporaba a un grado común de la escuela de referencia, el $25 \%$ se trasladaba con pase y el $15 \%$ restante era derivado a otra área (Padawer, 2010). En 2016 PGN atiende a 400 niños en $45 \mathrm{GN}$ de 31 escuelas. 
Revista de la Escuela de Ciencias de la Educación, año 14, nRo. 13, vol. 2, Julio a diciembre de 2018. Páginas 121-141. ISSN 1851-6297 (DESDE DICIEMBRE DE 2006 A DICIEMBRE DE 2017). ISSN 2362-3349 (EN LiNEA). MiRADAS A LAS ALTERNAtiVAS EDUCATIVAS AL FRACASO ESCOLAR. POLITICAS, EXPERIENCIAS, CONCEPTUALIZACIONES Y ESTUdIO DE CASO A INICIOS DEL S XXI. Cecilia Durantini Villarino.

El GN caso de estudio de la investigación doctoral pertenecía a una Escuela del cordón sur y diariamente funcionaba en el Centro Comunitario de una de las villas de emergencia de mayor tamaño de la ciudad. Los 12 niños del grupo llegaron con experiencias críticas a nivel familiar como las migraciones, el abandono parental, la delincuencia y adicciones de familiares próximos o su muerte por bandas barriales o violencia policial. Sufrieron el ingreso escolar tardío, repetición, deserciones y rechazo por parte de sus maestros, compañeros o directivos. Estos acontecimientos fragmentaron la continuidad de su existencia intensificando la vulnerabilidad asociada a las condiciones de vida.

El maestro, en su tercer año a cargo del GN, tenía formación docente en nivel inicial, primario y de adultos, y artística, experiencia profesional en las colonias infantiles municipales y el Proyecto Maestro+Maestro. Su discurso crítico de la escuela era sensible ante la estigmatización y los abusos de autoridad sobre niños y adolescentes en situaciones de exclusión social y educativa. Frente a ellas, defendía el reconocimiento y la confianza en los intereses y las posibilidades de aprender de estos niños.

\section{El diseño de investigación}

La investigación doctoral se encuadra epistemológicamente en las hipótesis de singularidad y oscuridad de lo real (Blanchard Laville, 1999), complejidad del objeto de estudio (Ardoino, 2005) y el conocer como la praxis de un sujeto social y de deseo (Castoriadis, 2007). A nivel metodológico opta por un estudio de caso descriptivo y en profundidad desde un enfoque clínico (Blanchard Laville, 1999; De Gaudelaj, 2008) e institucional (Fernández, 2006) con aportes de la etnografía (Rockwell, 2009). El diseño se organiza en ciclos de contrato con los sujetos, indagación diagnóstica, descripción analítica de los materiales, producción de avances, validación en reuniones de devolución, reformulación y reiteración del ciclo hasta el avance final.

El trabajo de 335 horas totales en terreno se desarrolla en tres fases. En el 2005 se entrevistan informantes claves, se contrata el diseño con el maestro y se prueban los instrumentos. Durante el año 2006 se efectúa la indagación sistemática en el GN caso y en el 2007, las reuniones de validación y devolución de los avances a los protagonistas.

Para la indagación se combinan observaciones de jornadas de clase y actividades especiales con audio grabación y registro escrito sistemático in situ, base de la escritura de crónicas. Se toman entrevistas abiertas a los actores y también entrevistas de análisis de las crónicas al maestro. Los niños participan en dispositivos expresivos y pruebas de rendimiento. Se recopilan documentos y producciones de los niños. A lo largo del trabajo,

El análisis conlleva un trabajo recursivo de lectura, descripción y construcción de categorías, generación de informes de base, descripciones analíticas (Rockwell, 2009), síntesis, periodizaciones, líneas de tiempo biográficas, gru- 
Revista de la Escuela de Ciencias de la Educación, año 14, nRo. 13, vol. 2, Julio a diciembre de 2018. Páginas $121-141$. ISSN 1851-6297 (DESDE DICIEMBRE DE 2006 A DICIEMBRE DE 2017). ISSN 2362-3349 (EN LínEA). MIRADAS A LAS ALTERNATIVAS EDUCATIVAS AL FRACASO ESCOLAR. POLITICAS, EXPERIENCIAS, CONCEPTUALIZACIONES Y ESTUDIO DE CASO A INICIOS DEL $S$ XXI. Cecilia Durantini VilLaRino.

pales y organizacionales, gráficos de las dinámicas y tramas vinculares, y su triangulación.

Las miradas interpretativa y comprensiva se complementan para reconstruir las lógicas de acción y captar los significados de la experiencia para los sujetos a nivel de lo dicho y permitido, lo silenciado o rumoreado, y lo negado u olvidado (Fernández, 2006) dentro de las tramas grupales y los procesos sociales e históricos de los que forman parte. A lo largo del trayecto se cuida la escucha empática, se suspenden los juicios evaluativos y prescriptivos, y se lleva un diario de reflexión sobre el proceso y de análisis de la implicación.

\section{GN caso como alternativa}

El estudio permite identificar una combinatoria de condiciones que activaron procesos de inclusión social y generaron oportunidades de vivir experiencias positivas en el grupo, favoreciendo el aprendizaje y el desarrollo de la confianza y autonomía en estos niños con experiencias escolares negativas. También muestra limitantes a la consolidación y profundización de estos logros. Aquí se abordan las posibilidades y las dificultades derivadas de la concreción singular que adoptaron el proyecto y las normas oficiales de PGN en el GN caso, en cuanto a su ubicación en el sistema educativo, la inserción en el Centro Comunitario y la articulación con la escuela. Los análisis se fundamentan empíricamente en los documentos normativos y en los sucesos y testimonios relevados, y teóricamente en las líneas institucionalistas expuestas.

\section{Las Posibilidades}

\section{Ubicación en el sistema educativo}

La Resolución de Creación de PGN establece que los GN pertenecen a las escuelas y asigna competencia y responsabilidad a dos líneas de autoridad dependientes de la Dirección de Educación Primaria. Una corresponde al Equipo Técnico de PGN y la otra, a las supervisiones de distrito y las conducciones de las escuelas comunes donde dichos GN funcionan. La normativa no regula ni prevé una reglamentación que determine los límites entre estas instancias.

El material del GN caso muestra la potencialidad de esta distribución paralela cuando las autoridades están comprometidas con el proyecto, tienen capacidad para la toma consensuada de decisiones y desarrollan una modalidad de gestión colaborativa.

El supervisor se ocupó personalmente de la elección de la escuela para la apertura del primer GN en su distrito, y de convocar al maestro por su experiencia en el Programa Zap, y por la creatividad y seriedad en el desempeño. El director de la Escuela, con más de 20 años de trayectoria en el barrio, gestionó el aula en el Centro Comunitario donde visitaba con frecuencia al grupo 
Revista de la Escuela de Ciencias de la Educación, año 14, nRo. 13, vol. 2, Julio a diciembre de 2018. Páginas 121-141. ISSN 1851-6297 (DESDE DICIEMBRE DE 2006 A DICIEMBRE DE 2017). ISSN 2362-3349 (EN LiNEA). MiRADAS A LAS ALTERNATIVAS EDUCATIVAS AL FRACASO ESCOLAR. POLITICAS, EXPERIENCIAS, CONCEPTUALIZACIONES Y ESTUDIO DE CASO A INICIOS DEL $S$ XXI. Cecilia Durantini Villarino.

y promovió la articulación con el establecimiento. El primer coordinador de PGN acompañó al maestro durante sus primeros tiempos. El maestro reconoció ampliamente a estas figuras por su preocupación genuina por los niños, responsabilidad en la tarea y valentía para transgredir la burocratización del sistema. De sus dichos, se infiere que ambos se han constituido en modelos y fuentes de legitimación de su estilo de enseñanza (Cols, 2011).

\section{Inserción en el Centro Comunitario}

El plan de acción define como meta ofrecer condiciones institucionales y pedagógicas para garantizar, en el menor tiempo posible, la inserción escolar de la población destinataria. Entre ellas, la aproximación a los lugares no convencionales para el sistema educativo pero que constituyen contextos de referencia para los niños.

El GN caso comenzó a funcionar en un comedor comunitario que no reunía las condiciones edilicias mínimas para dar clase. Al año siguiente, el director de la Escuela logró un convenio con el Centro Comunitario barrial, que designaba un aula para el funcionamiento diario del GN.

El coordinador de PGN lo recordaba

El supervisor enseguida dijo este director ideal... El director es macanudo, GN está en esa escuela porque él conoce a todos los referentes de la villa, en otra escuela no hubiera funcionado. Se preocupa mucho... está muy comprometido con la escuela, con el GN, es de este barrio... dijo 'yo les voy a buscar otro lugar, en el comedor no va más', el comedor era un galpón con guardería para bebés, no había aula, ruido todo el día, se morían de frío en invierno, poca luz. Él fue... habló, si no hubiese sido por el director ahí no entrábamos para nada (coordinador, entrevista 2004).

Allí los niños accedían a recursos para las actividades especiales, participaban en propuestas artísticas y deportivas como muestras de cine y talleres de música y presenciaban gimnasia para abuelos y murga para adolescentes. El maestro tomaba bien uno o varios momentos de descanso y recreación, con juegos didácticos o en el patio con juegos deportivos.

Según los análisis efectuados, este tipo de localización facilita el desarrollo de experiencias cuya continuidad en el tiempo sienta las bases para que los niños confíen, cuiden y se apropien del espacio educativo. También permite al maestro adaptar las regulaciones de tiempo y espacio al ritmo de trabajo y clima grupal. Además, el grupo puede tomar contacto con otras formas de organización de las actividades culturales en las que sujetos de distintas edades enseñan y aprenden diferentes tipos de conocimientos de maneras diferentes. 
Revista de la Escuela de Ciencias de la Educación, año 14, nRo. 13, vol. 2, Julio a diciembre de 2018. Páginas $121-141$. ISSN 1851-6297 (DESDE DICIEMBRE DE 2006 A DICIEMBRE DE 2017). ISSN 2362-3349 (EN LíNEA). MIRADAS A LAS ALTERNATIVAS EDUCATIVAS AL FRACASO ESCOLAR. POLITICAS, EXPERIENCIAS, CONCEPTUALIZACIONES Y ESTUDIO DE CASO A INICIOS DEL $S$ XXI. Cecilia Durantini VilLaRino.

\section{Articulación con la Escuela}

Los documentos no explicitan la idea de articulación institucional aunque plantean elementos asociados. La meta del proyecto permite enmarcarla como una de las condiciones a ofrecer para que los GN actúen como "puentes" de reinserción escolar. La norma identifica a todos los GN como "dependientes" del establecimiento de pertenencia. Con lo cual, al inscribirse en un $\mathrm{GN}$, los niños ingresan o se reincorporan al sistema educativo como alumnos de la escuela responsable por su trayectoria.

EI GN caso desarrolló diferentes actividades de articulación con la escuela. Los niños asistían una vez por semana a las clases de matemática, prácticas del lenguaje, música o dibujo de los grados comunes. Ese día trabajaban en los proyectos del GN integrados con biblioteca e informática como "Pintores contemporáneos", "Este soy yo". El grupo participaba, además, de actos y excursiones. El maestro, por su parte, daba apoyo a los niños reinsertados.

Mediante estas modalidades, los niños pudieron vincularse con docentes, auxiliares y compañeros de la escuela, a quienes recurrieron en ausencia del maestro o en el retorno a la escuela, generar productos concretos en el marco de los proyectos, dramatizar una obra el día del maestro y comprar sus propios libros en la Feria del Libro.

\section{Las Limitaciones}

\section{Ubicación en el sistema educativo}

La doble ubicación organizacional de los GN está reglamentada en dos artículos. Uno indica que el director de la Escuela y el maestro del GN deben elaborar un informe con el grado de destino de los niños y recomendaciones para garantizar su permanencia. No menciona al Equipo de PGN ni prevé dispositivos para el acompañamiento y el seguimiento de los niños en los grados comunes. El otro artículo encomienda la conformación de una Comisión Especial para elaborar los criterios de promoción, evaluación y acreditación de PGN y las adecuaciones al Reglamento y la documentación escolar para incorporar a los alumnos de los GN. Durante su breve funcionamiento, la Comisión no llegó a producir estos instrumentos.

Los vacíos en la distribución de responsabilidades y competencias entre PGN y las escuelas en cuestiones claves como la reinserción de los niños, se jugaron dramáticamente para el GN caso.

La primera coordinación de PGN apuntaba a finalizar el proyecto por el cumplimiento de los objetivos, oponiéndose a su consolidación como una oferta estructural. La coordinación sucesora apostó al crecimiento del proyecto lo cual requería matrícula y éxito en su reinserción. En esos años, la conducción de la Escuela viraba desde el modelo del anterior director, basado en la inclusión y la atención prioritaria a los grupos vulnerables, hacia uno de mejora de la calidad educativa por la selección meritocrática. Con lo cual, la búsqueda 
Revista de la Escuela de Ciencias de la Educación, año 14, nRo. 13, vol. 2, Julio a diciembre de 2018. Páginas 121-141. ISSN 1851-6297 (DESDE DICIEMBRE DE 2006 A DICIEMBRE DE 2017). ISSN 2362-3349 (EN LiNEA). MiRADAS A LAS ALTERNAtiVAS EDUCATIVAS AL FRACASO ESCOLAR. POLITICAS, EXPERIENCIAS, CONCEPTUALIZACIONES Y ESTUdIO DE CASO A INICIOS DEL S XXI. Cecilia Durantini Villarino.

de buenos resultados de PGN en términos de alumnos en GN e integrados a grados comunes se contrapuso a la lógica de la Escuela.

Al cierre del ciclo 2006, la directora requirió pruebas escritas a los niños de GN. El maestro las presentó con un informe que recomendaba la permanencia de algunos niños en el GN y la incorporación de otros a un grado común, en base a la trayectoria, el nivel de aprendizaje, socialización y desarrollo de la autonomía. La puja entre la directora que rechazó la reinserción de los niños y la coordinación de PGN que presionaba por el retorno de todos, se resolvió por acuerdo entre ambas autoridades. La directora aceptó firmar la promoción al grado común de aquellos niños con pase a otro establecimiento. Para reinsertarse en su escuela, debían mostrar el nivel de rendimiento exigido en los turnos de examen previstos. En 2007, dos niños se reinsertaron fuera y una niña en un grado común de la misma.

\section{Inserción en el Centro Comunitario}

La normativa define la inserción territorial de los GN en cuanto a su localización y a los modos de vinculación con otros actores sociales. Los objetivos específicos apuntan al trabajo directo con las familias sobre la importancia de la educación formal y a la formación de redes con referentes y agrupaciones comunitarias, organizaciones gubernamentales y no gubernamentales.

La localización de GN en lugares de referencia para los niños se dirige a facilitar la permanencia de aquellos con mayor resistencia a la escuela y el retorno a la misma. La normativa lista las características de los locales para garantizar la seguridad, tarea y recreación de los niños y el maestro. Sin embargo, el articulado siguiente establece que el funcionamiento fuera de la escuela reviste un carácter transitorio y debe efectuarse un traslado definitivo a la misma para contribuir a la socialización escolar y a la reinserción en los grados comunes.

La ambigüedad de la norma en este punto parece informar, según los análisis, sobre los núcleos pre-institucionales integrados en PGN. Ulloa (1969) plantea que en toda organización se integran núcleos constituidos por proyectos afines bajo un proyecto común con un régimen de normas compartidas. De estos núcleos surgen especializaciones que tienden al crecimiento o dispersión de la organización. El predominio de una u otra tendencia se asocia al grado de explicitación de las diferencias existentes entre ellos. El silenciamiento y negación de desacuerdos importantes suele manifestarse en la estructuración de divisiones y modalidades funcionales como mutuamente excluyentes. Los grupos que las representan se oponen de manera irreconciliable mientras que los reales antagonismos permanecen no dichos. Con lo cual se enquistan repitiéndose bajo sucesivas versiones en la historia de la organización.

Los relatos de la historia de PGN narran la inserción en los barrios como una estrategia exitosa frente a los niños que abandonaron la asistencia al 
Revista de la Escuela de Ciencias de la Educación, año 14, nRo. 13, vol. 2, Julio a diciembre de 2018. Páginas $121-141$. ISSN 1851-6297 (DESDE DICIEMBRE DE 2006 A DICIEMBRE DE 2017). ISSN 2362-3349 (EN LínEA). MIRADAS A LAS ALTERNATIVAS EDUCATIVAS AL FRACASO ESCOLAR. POLITICAS, EXPERIENCIAS, CONCEPTUALIZACIONES Y ESTUDIO DE CASO A INICIOS DEL $S$ XXI. Cecilia Durantini VilLaRino.

primer GN en una escuela, lo cual motivó su recomendación con las instituciones particularmente reactivas al proyecto. La nueva coordinación, en cambio, fomentó el traslado a los establecimientos escolares, donde actualmente se cubre la mayor parte de la oferta.

Como se vio, gracias a la gestión del director, el GN caso se trasladó de un comedor en deficiente estado al aula en el Centro Comunitario. Los días de articulación con la Escuela, el GN funcionó en un patio o en la biblioteca hasta que el director habilitó un aula depósito. La nueva conducción recuperó esta aula para tales fines y efectuó el traslado de GN al edificio. El grupo fue ubicado en el laboratorio, un salón con banquetas que debían desocupar cuando otros grados realizaban prácticas para reubicarse en los lugares vacantes según el día. El maestro lo significó como una pérdida para la continuidad de los procesos de aprendizaje y desarrollo de los niños. Desde la crítica a la cultura escolar, defendía la localización en el Centro Comunitario en base a la normativa, la posibilidad de adaptación y buen clima de trabajo, y las ventajas comparativas a otros proyectos.

GN funciona mucho mejor acá que en la escuela... La escuela, la conducta, los líos, los alborota demasiado... Los recreos son fuertes, pesados, se pegan, violentos. Acá funcionan más tranquilos, juegan a la pelota... con los juegos de mesa. El ritmo de allá no les permite eso. Aparte la idea del GN era que esté adentro de la villa para recuperar a los pibes...En comparación a los grados de recuperación, y el proyecto Zap, al estar en la escuela, el ambiente hace que te termines enviciando... rutinas que hacen a una vorágine de locura (maestro GN, entrevista 2006).

En la historia del GN caso, la inserción resurge como una cuestión controversial cuyas resoluciones parecen asociarse al posicionamiento político pedagógico de la conducción escolar respecto a la integración de los niños excluidos, y con el modelo pedagógico y de vinculación institucional de la coordinación de PGN.

\section{Articulación con la Escuela}

Si bien los documentos no presentan una línea de articulación institucional, aunque remiten a ella, los actores del GN caso la evocan reiteradamente con diferente valoración y significado. El primer coordinador la subordinaba a la receptividad y adaptación de la escuela y los docentes. La asistente técnica la consideraba un medio de socialización clave para el retorno exitoso a la escuela. Las maestras de la Escuela coincidieron en su potencial como en la evaluación negativa de su implementación. En general planteaban que la articulación era una ficción marcada por la baja participación en las actividades, la escasa interacción con los niños del establecimiento y la discontinuidad de las clases con el grupo, debido a paros, actos, jornadas y ausencias propias o de los niños. No obstante, algunas docentes valoraban las oportunidades de 
Revista de la Escuela de Ciencias de la Educación, año 14, nRo. 13, vol. 2, Julio a diciembre de 2018. Páginas 121-141. ISSN 1851-6297 (DESDE DICIEMBRE DE 2006 A DICIEMBRE DE 2017). ISSN 2362-3349 (EN LiNEA). MiRADAS A LAS ALTERNATIVAS EDUCATIVAS AL FRACASO ESCOLAR. POLITICAS, EXPERIENCIAS, CONCEPTUALIZACIONES Y ESTUDIO DE CASO A INICIOS DEL $S$ XXI. Cecilia Durantini Villarino.

aprendizaje que permitía ofrecer.

La frecuencia y modalidades de articulación con la Escuela se redujeron a través del tiempo. En el año 2004, los niños asistían directamente a la Escuela dos veces por semana. Una para las clases de música, dibujo e inglés en el grado común de destino, y otra para el desarrollo de los proyectos integrados. En el año 2006, el GN se reunía en el Centro Comunitario para asistir juntos a la Escuela y evitar las quejas del personal por el cuidado de los niños. El día de articulación, el grupo trabajaba en el proyecto integrado y asistía a las clases de música y de dibujo. Una tarde, el profesor de dibujo colocó a uno de los niños en penitencia frente al grupo de 30 alumnos. Frente a este hecho, el maestro del GN caso experimentó una fuerte vulnerabilidad y falta de reconocimiento y sostén institucional al grupo.

Encima la escuela al no estar abierta al proyecto... era como que les estaba debiendo un favor, una molestia y es el trabajo de ellos... las notas nunca me las pasaban, como que lo incorporaban al nene y lo tenían ahí... Cuando encontré a Cristian en penitencia, le planteé a dirección que no podían seguir con el profesor de dibujo, la directora no intervino, no mediaron, me sentí muy solo... los chicos son parte de la institución, GN no es mi escuela privada, yo dependo de un lugar y la escuela tiene que hacerse cargo (maestro GN, entrevista 2006)

El impacto negativo sobre los niños se manifestó en el ausentismo el día de articulación, la expresión abierta del rechazo a la escuela y del gusto por los actos, excursiones y proyectos integrados con informática.

\section{Posibilidades y límites del GN caso}

En cada GN las definiciones normativas en cuanto a la ubicación en el sistema educativo, la inserción territorial y la articulación institucional con la escuela se concretan de diferentes formas.

EI GN caso se hallaba en un distrito escolar históricamente comprometido con los sectores populares donde contaba con el acompañamiento activo del director de la Escuela, alineado a esta tradición. En ese marco, estaban garantizadas las experiencias de articulación institucional y la inserción en un Centro Comunitario dispuesto a albergar al grupo. El maestro se apoyó en esta pertenencia múltiple y en la doble línea de autoridades para ampliar los márgenes de autonomía del GN.

Según los análisis, la singular combinatoria de estos elementos habilita la configuración de un espacio social de emplazamiento de GN, identificado como una zona porosa entre dinámicas institucionales y movimientos educativos (Durantini, 2008). Esta zona opera como una condición clave para el ingreso y la permanencia de los niños en la respuesta educativa y con ella, en el sistema. También para la inclusión social pues se activan procesos de filiación psicosocial de los niños y sus familias a una red de la se hallan exclui- 
Revista de la Escuela de Ciencias de la Educación, año 14, nRo. 13, vol. 2, Julio a diciembre de 2018. Páginas $121-141$. ISSN 1851-6297 (DESDE DICIEMBRE DE 2006 A DICIEMBRE DE 2017). ISSN 2362-3349 (EN LínEA). MIRADAS A LAS ALTERNATIVAS EDUCATIVAS AL FRACASO ESCOLAR. POLITICAS, EXPERIENCIAS, CONCEPTUALIZACIONES Y ESTUDIO DE CASO A INICIOS DEL $S$ XXI. Cecilia Durantini VilLaRino.

dos. Con lo cual, se amplía su acceso a las actividades, servicios y recursos disponibles.

La mitad de los niños de GN tramitó por primera vez su documento nacional de identidad, las madres solicitaron o aconsejaron a vecinas asesoría legal, meriendas, becas escolares, guardapolvos y útiles, campañas de vacunación, etc. Al tiempo que se redujeron sus caídas al ser derivados de una instancia a otra según la demanda en juego. Por ejemplo, el portero del Centro Comunitario acompañó al maestro a la vivienda de un niño con inasistencias reiteradas para prevenir una nueva deserción, la pediatra del Centro de Salud asistió la inscripción en GN de un niño atendido por bajo peso, las maestras de cuarto grado invitaron al GN a sumarse en la excursión al musical Ópera Pampa, la profesora de informática llamó al maestro cuando el grupo faltó dos semanas consecutivas al establecimiento, las familias de niños ya integrados a la escuela comunicaron a sus vecinas la buena experiencia en GN como espacio para lograrlo.

Es decir que, en esta red se entraman, junto a la oferta regular de las organizaciones educativas, sanitarias y sociales, un tejido de relaciones de proximidad anudado entre los miembros de las organizaciones protagonistas, los referentes de la comunidad y las familias y vecinos. Las múltiples interacciones entre ellos parecen potenciar la activación de "lo social" (Castel, 2004) entendido como "el conjunto de dispositivos puestos en marcha para compensar el déficit de recursos necesarios para vivir en sociedad por los propios medios" (p. 28).

La reconstrucción de la dinámica educativa en esta zona porosa muestra que el grupo de GN transitó por una diversidad de formas del campo cultural que posibilitaron a los niños desde compartir un ciclo de cine abierto en el Centro Comunitario hasta dramatizar una obra frente al público de docentes, padres y alumnos de la escuela y a los aplausos de sus familias. Tanto participar en un juego de cuentos tradicionales en la escuela como en una fiesta del Centro Comunitario donde un adolescente interpretó temas de rock con su guitarra y un grupo de abuelos presentó destrezas físicas al cierre del ciclo lectivo.

Se entiende que este tipo de acontecimientos constituyen para los niños, oportunidades de vivir experiencias de creación, transmisión y filiación comunitaria e intergeneracional, y desarrollo de proyectos y defensa de la dignidad de la persona, sostenidas sobre lazos colectivos y grupales en resistencia a la violencia y la exclusión. Ellas describen la potencialidad educativa alternativa profunda de un espacio como el GN.

En palabras de la mamá de dos niñas del grupo que habían abandonado la escuela

Eso es lo único bueno que me pasó este año, lo bien que están mis hijas. La energía que tienen... En la otra escuela eran muy cerradas, se aisla- 
Revista de la Escuela de Ciencias de la Educación, año 14, nRo. 13, vol. 2, Julio a diciembre de 2018. Páginas 121-141. ISSN 1851-6297 (DESDE DICIEMBRE DE 2006 A DICIEMBRE DE 2017). ISSN 2362-3349 (EN LiNEA). MiRADAS A LAS ALTERNAtiVAs educativas al fRACASO ESCOLAR. POLITICAS, EXPERIENCIAS, CONCEPTUALIZACIONES Y ESTUdIO DE CASO A INICIOS DEL $S$ XXI. Cecilia Durantini Villarino.

ban, les caía una lágrima... pero acá están más sanas, tienen más ganas de hacer cosas... se tienen más confianza... se contactan con los otros chicos. Camila dice me quiero sacar un muy bien 10, se pone las pilas y estudia... sé que en el GN mis hijas están en buenas manos, el maestro las cuida... si ellas están tristes, les pregunta qué te pasa. Te manda llamar... Los maestros eso no lo hacen... es una persona que tiene paciencia, muy buena, que las supo ayudar, les habla, que lo quieren... Yo quiero que cambie todo, que haya más justicia, que los chicos no estén al margen de la muerte en cada paso que den. Que la muerte de mi hijo con la policía, no le pase a nadie, si algún chico necesita de un apoyo para aprender, para salir adelante, yo creo que el GN es apto para eso (madre GN, entrevista 2004).

En la exposición se plantea que la normativa y proyecto oficiales del PGN revisten insuficiencias respecto a la distribución de responsabilidades y competencias entre PGN y las escuelas, ambigüedad en cuanto a la localización de GN en lugares no convencionales para el sistema, y ausencias de reglamentación sobre las formas y espacios concretos de articulación institucional. De ellas se derivan dificultades más o menos estructurales para el funcionamiento de los GN, plausibles de mejora bajo una revisión y reformulación crítica del proyecto y los documentos.

En el GN caso, estas debilidades se conjugaron críticamente, por una parte, con las tensiones entre las culturas y lógicas de funcionamiento de las organizaciones, la resistencia a revisar los diagnósticos, formatos y modos de trabajo establecidos y la falta de instancias y canales de comunicación entre los actores directamente afectados al proyecto.

Por otra, se sumaron los cambios asumidos por las nuevas autoridades de PGN y de la Escuela en cuanto a la posición política pedagógica frente a la situación de exclusión de los niños, el enfoque de cumplimiento del proyecto y el estilo de negociación con otras organizaciones.

Las maestras señalaron supuestos umbrales de aprendizaje en los niños, condicionantes sociales y familiares insuperables en un contexto de escasez y disfunción institucional. Frente a lo cual, visualizaron opciones como la finalización de la escolaridad en ámbitos paralelos, la incorporación inmediata a grados comunes no numerosos, el trabajo en aulas separadas o la articulación con áreas curriculares fuera del establecimiento.

Las familias valoraban ampliamente los avances logrados, pero ansiaban una trayectoria escolar "normal como el resto de los niños" para sus hijos. Algunos niños del GN caso expresaron interés por estar en la escuela, "pasar de grado", seguir aprendiendo. Otros manifestaron su rechazo a la escuela y preferencia por continuar en el GN más agradable, ordenado y tranquilo junto al maestro que los escuchaba, atendía y ayudaba.

Las dificultades analizadas convergen alrededor de la cuestión de la presencia, léase, visibilidad y participación, de los niños ya excluidos-expulsados, 
Revista de la Escuela de Ciencias de la Educación, año 14, nRo. 13, vol. 2, Julio a diciembre de 2018. Páginas $121-141$. ISSN 1851-6297 (DESDE DICIEMBRE DE 2006 A DICIEMBRE DE 2017). ISSN 2362-3349 (EN LínEA). MIRADAS A LAS ALTERNATIVAS EDUCATIVAS AL FRACASO ESCOLAR. POLITICAS, EXPERIENCIAS, CONCEPTUALIZACIONES Y ESTUDIO DE CASO A INICIOS DEL $S$ XXI. Cecilia Durantini VilLaRino.

en su retorno a la escuela común. Por consiguiente, alrededor del problema de su lugar y tratamiento. Esta cuestión remite a la acuciante pregunta por qué hacer, dónde colocar, cómo convertir a los "restos" vivientes del fracaso institucional, social y personal del proyecto de igualdad e inclusión. En este escenario, cabe plantear si las alternativas educativas sirven fundamentalmente al clivaje (Bleger, 2004) entre los "fracasados escolares" y los "alumnos normales" y "exitosos". En tal caso, como movimiento transformador deberían confrontar a las tendencias de desactivación y recuperación encarnadas en las prácticas y significaciones conservadoras.

\section{A modo de cierre}

Las políticas educativas de la primera década privilegiaron la atención del problema de la exclusión en el cumplimiento del derecho a la educación. Entre las líneas para la democratización educativa, la mejora de los aprendizajes y la recuperación del sentido de la experiencia, las propuestas alternativas pusieron en el horizonte la cuestión del cambio de algún aspecto del formato escolar clásico hasta la institución misma.

Su uso como analizadores (Lourau, 1970), es decir catalizadores de las divisiones y significaciones sociales inmovilizadas y naturalizadas que regulan la acción de los sujetos y la atribución de sentido a su experiencia, aumenta la visibilidad de los mecanismos de exclusión y los obstáculos institucionales al cambio en los niveles meso y micro de funcionamiento del sistema. Así como evidencia la distinción entre los límites del formato escolar convencional para responder creativamente a este problema, de los límites que imponen las causas socioeconómicas del fracaso y exceden largamente a la intervención escolar.

Lo cual no debería conducir al abandono de la inclusión de todos los niños como horizonte posible. Por el contrario, compromete aún más la pregunta sobre el potencial de las alternativas, y de las instituciones educativas actuales, para desarrollar condiciones que habiliten el aprender y el acceso al conocimiento; las experiencias de aprendizaje con sentido para sí y junto a otros; la integración y participación activas en la vida institucional y comunitaria.

\section{Referencias bibliográficas}

- Agambem, G. (2003). Estado de excepción. Homo sacer II. 1. Buenos Aires: Adriana Hidalgo Editora.

- Ardoino, J. (2005). Pensar la educación desde una perspectiva epistemológica. Buenos Aires: Novedades educativas

- Baquero, R. (2006). Sujetos y aprendizaje. Buenos Aires: Ministerio de Educación, Ciencia y Tecnología de la Nación.

- Blanchard Laville, C. (1999). L'approche clinique d'inspiration psychanalytique: enjeux théoriques et méthodologiques. Revue Française de Pédagogie, 127, pp. 111-162.

- Bleger, J. (2002). El grupo como institución y el grupo en las instituciones. En Kaës, R 
Revista de la Escuela de Ciencias de la Educación, año 14, nRo. 13, vol. 2, Julio a diciembre de 2018. Páginas 121-141. ISSN 1851-6297 (DESDE DICIEMBRE DE 2006 A DICIEMBRE DE 2017). ISSN 2362-3349 (EN LiNEA). MiRADAS A LAS ALTERNAtIVAS EDUCATIVAS AL FRACASO ESCOLAR. POLITICAS, EXPERIENCIAS, CONCEPTUALIZACIONES Y ESTUDIO DE CASO A INICIOS DEL $S$ XXI. Cecilia Durantini Villarino.

(Ed). La institución y las instituciones. Buenos Aires: Paidós. pp 68-83.

- Bleger, J. (2004). Psicohigiene y Psicología institucional. Buenos Aires: Paidós

- Castel, R. (2004). La inseguridad social. ¿Qué es estar protegido? Buenos Aires: Manantial.

- Castoriadis, C (2007). La institución imaginaria de la sociedad. Buenos Aires: Tusquets

- Cols, E (2011). Estilos de enseñanza. Sentidos personales y configuraciones de acción tras la semejanza de las palabras. Rosario: Homo Sapiens.

- De Gaudelaj, V. (2008). Las fuentes de la vergüenza. Buenos Aires: Mármol Izquierdo.

- Dubet, F. (2002). El declive de la institución. Profesiones, sujetos e individuos ante la reforma del estado. Barcelona: Gedisa.

- Dubet, F. (2007). La experiencia sociológica. Buenos Aires: Gedisa.

- Durantini Villarino, C. (2004). Proyectos generados en respuesta a condiciones críticas. Investigadora Becaria en Formación 2003-2004. Instituto de Investigaciones en Ciencias de la Educación. Facultad de Filosofía y Letras. Universidad de Buenos Aires. Proyecto Ubacyt. Directora Fernández, Lidia.

- Durantini Villarino, C. (2006). Proyectos generados en respuesta a condiciones críticas: El caso del Grado de Nivelación. En Revista del Instituto de Investigaciones de Ciencias de la Educación, Universidad de Buenos Aires, 24, pp.11-19.

- Durantini Villarino, C. (2008a). Acerca de lo imaginado y lo por imaginar. Concepciones de los actores involucrados en una respuesta posible al fracaso escolar. En Revista de la Escuela de Ciencias de la Educación, Universidad Nacional de Rosario, 3, pp 155-178.

- Durantini Villarino, C. (2008b). Espacios Educativos Alternativos y "fracaso escolar": acerca de los posibles en una zona porosa de localización. En Revista Praxis del Instituto de Ciencias de la Educación para la Investigación Interdisciplinaria, Universidad Nacional de La Pampa, 12, pp. 22-28.

- Durantini Villarino, C. (2009). El Porvenir de una llusión, Actas Primeras Jornadas Nacionales Los enfoques institucionales en educación. En Red de Estudios Institucionales en Educación, pp. 51-78.

- Durantini Villarino, C. (2010). Institución y organizaciones de la educación: las limitaciones a los atisbos de cambio frente al problema del "fracaso escolar". En Revista Educación, Sociedad y Lenguaje, Universidad Nacional de la Pampa, 7, pp 79-100.

- Durantini Villarino, C. (2011). Enseñar o cuidar a niños maltratados socialmente: el procesamiento de este dilema en el maestro de un espacio educativo alternativo. En Revista de la Escuela de Ciencias de la Educación, Universidad Nacional de Rosario, 6, pp. 197-212

- Durantini Villarino, C. (2014). Tesis doctoral Espacios Educativos Alternativos. Acerca de donde la educación imposible se hace posible. Facultad de Filosofía y Letras. Universidad de Buenos Aires. Beca Doctoral 2005-2009. Instituto de Investigaciones en Ciencias de la Educación, Facultad de Filosofía y Letras. Universidad de Buenos Aires. Proyecto Ubacyt Directora, Fernández Lidia.

- Durantini Villarino, C. (2014). Un espacio alternativo donde estar y aprender: niños con experiencias de fracaso escolar construyendo su aula en un Centro Comunitario. En Revista Praxis del Instituto de Ciencias de la Educación para la Investigación 
Revista de la Escuela de Ciencias de la Educación, año 14, nRo. 13, vol. 2, Julio a diciembre de 2018. Páginas $121-141$. ISSN 1851-6297 (DESDE DICIEMBRE DE 2006 A DICIEMBRE DE 2017). ISSN 2362-3349 (EN LíNEA). MIRADAS A LAS ALTERNATIVAS EDUCATIVAS AL FRACASO ESCOLAR. POLITICAS, EXPERIENCIAS, CONCEPTUALIZACIONES Y ESTUDIO DE CASO A INICIOS DEL $S$ XXI. Cecilia Durantini VilLarino.

Interdisciplinaria, Universidad Nacional de la Pampa, Argentina, 18, pp. 102-110.

- Enriquez, E. (1992). Educación y Formación. Aportes desde una teoría de la institución y las organizaciones. Buenos Aires: Novedades educativas.

- Enriquez, E. (2002). La institución y la organización en la educación y la formación. Buenos Aires: Novedades Educativas.

- Fernández, L. M. (1994). Instituciones Educativas. Buenos Aires: Paidós.

- Fernández, L. M. (2006). "Espacios institucionalizados de la educación. Algunos componentes nucleares en la identidad institucional y su consecuencia para el análisis". En Landesmann (Ed.) Instituciones Educativas: Instituyendo disciplinas e identidades. México: Casa Juan Pablos. pp. 29-60.

- Ferreira, F.H.G., (2013). Economic mobility and the rise of the Latin American middle class. Washington, Banco Mundial. [Extraído el 10 de marzo de 2016 de https:// openknowledge.worldbank.org/bitstream/handle/10986/11858/9780821396346.pdf].

- Lobrot, M. (1974). Pedagogía institucional. La escuela hacia la autogestión. Buenos Aires: Humanitas.

- Loureau, R. (2001). Análisis institucional. Buenos Aires: Amorrortu.

- Padawer, A. (2010). La enseñanza primaria en contextos de desigualdad social y diversidad cultural. Estudios sobre políticas de fracaso escolar en escuelas de educación común. Ciudad de Buenos Aires: Ministerio de Educación.

- Poggi, M. (2014). La educación en América Latina: logros y desafíos pendientes. Buenos Aires: Santillana.

- Resolución de Creación del Proyecto GN Nº 2409/03, Ministerio de Educación, Ciudad de Buenos Aires

- Rivas, A. (2015). América Latina después de PISA: lecciones aprendidas de la educación en siete países 2000-2015. Buenos Aires: Fundación CIPPEC. [Extraído el 7 de marzo de 2016 de http://cippec.org/mapeal/wp-content/uploads/2015/05/ Rivas_A_2015_America_Latina_despues_de_PISA.pdf].

- Rockwell, E. (2009). La experiencia etnográfica. Buenos Aires: Paidós.

- Siteal, (2011). El desafío de universalizar el nivel primario [Extraído el 10 de octubre de 2015 de http://www.siteal.iipeoei.org/sites/default/files/siteal_destacado_11042011. pdf].

- Tedesco, J.C. (2012). Educación y Justicia Social en América Latina. Buenos Aires: Fondo de Cultura Económica.

- Tenti Fanfani, E. (2001). El rendimiento escolar en la Argentina. Análisis de resultados y factores. Buenos Aires: Losada.

- Tenti Fanfani, E. (2007). La escuela y la cuestión social. Ensayos de sociología de la educación. Buenos Aires: Siglo XXI.

- Terigi, F. (2009). Segmentación urbana y educación en América Latina. Los retos de la inclusión. Madrid, OEI. [Extraído el 16 de abril de 2016 de http://www.rinace.net/reice/ numeros/arts/vol7num4/REICE\%207,4.pdf].

- Trilla, J. (2005). El legado pedagógico del siglo XX para la escuela del siglo XXI. Barcelona: Graó.

- Ulloa, F. (1969). Psicología de las instituciones. Una aproximación psicoanalítica. En Revista APA. Buenos Aires. Tomo XXVI. 
Revista de la Escuela de Ciencias de la Educación, año 14, nRo. 13, vol. 2, julio a diciembre de 2018. Páginas $121-141$. ISSN 1851-6297 (DESDE DICIEMBRE DE 2006 A DICIEMBRE DE 2017). ISSN 2362-3349 (EN LiNEA). MiRADAS A LAS ALTERNATIVAS EDUCATIVAS AL FRACASO ESCOLAR. POLITICAS, EXPERIENCIAS, CONCEPTUALIZACIONES Y ESTUDIO DE CASO A INICIOS DEL $S$ XXI. Cecilia Durantini Villarino.

- UNESCO-OREALC. (2008). Los Aprendizajes de los Estudiantes de América Latina y el Caribe. Primer reporte de los resultados del Segundo Estudio Regional Comparativo y Explicativo. Santiago: Oficina Regional de Educación para América Latina y el Caribe de la UNESCO, Laboratorio Latinoamericano de Evaluación de la Calidad de la Educación [Extraído el 14 de febrero de 2016 de http://unesdoc.unesco.org/ images/0016/001606/160660s.pdf]. 